\title{
NB-IoT Micro-Operator for Smart Campus: Performance and Lessons Learned in 5GTN
}

\author{
Rumana Yasmin \\ Centre for Wireless Communications \\ University of Oulu \\ Oulu, Finland \\ rumana.yasmin@oulu.fi \\ Ari Pouttu \\ Centre for Wireless Communications \\ University of Oulu \\ Oulu, Finland \\ ari.pouttu@oulu.fi
}

\author{
Konstantin Mikhaylov \\ Centre for Wireless Communications \\ University of Oulu \\ Oulu, Finland \\ konstantin.mikhaylov@oulu.fi \\ Ville Niemelä \\ Centre for Wireless Communications \\ University of Oulu \\ Oulu, Finland \\ ville.niemela@oulu.fi
}

\author{
Muhammad Arif \\ Centre for Wireless Communications \\ University of Oulu \\ Oulu, Finland \\ muhammad.arif@oulu.fi \\ Olli Liinamaa \\ Centre for Wireless Communications \\ University of Oulu \\ Oulu, Finland \\ olli.liinamaa@oulu.fi
}

\begin{abstract}
In recent years, many new radio-based connectivity solutions for the Internet of Things (IoT) have been proposed. At the same time, development towards the $6 \mathrm{G}$ has brought on the stage the new business concepts. One of them is the concept of a micro-operator and implies the local entities to act as the telecom infrastructure owner and provider in their premises. In the current paper, we discuss the deployment and report the practical performance of a single-cell NB-IoT deployed as a part of the 5G Test Network (5GTN) and controlled by a smart-campus micro-operator. The practical measurements reported in the paper have been carried in the University of Oulu within a huge interconnected indoor environment with the total floor area of $188600 \mathrm{~m}^{2}$. Our results demonstrate that the NB-IoT technology is a viable connectivity solution for various non-critical machine-based applications deployed indoors, highlight the practical performance of this technology, and reveal some practical specifics and challenges of acting as an IoT micro-operator.
\end{abstract}

Keywords - NB-IoT, micro-operator, 5GTN, Smart campus, Internet-of-Things, MTC, LPWAN, 6GFlagship

\section{INTRODUCTION}

Myriads of the miniature autonomous devices take their place around us every single day, making a decisive step towards the formation of the long-talked-about Internet-ofThings (IoT). The concept of IoT has enormous potential to change the world we live in, work, and study in the context of multiple different verticals. In this paper, we focus primarily on one of these verticals, namely the smart campus.

For many years the campuses of colleges and universities have been the centers of innovations, educating the experts and driving both the methodology and the technology development forth. In this context, the concept of smart campus aims at enabling the novel, innovative ecosystem, based on the integration of the cutting-edge information and communication paradigms [1]. The goal of a smart-campus goes well beyond providing the services for the students' and staff's comfort, or the efficient utilization of the resources. Instead, a smart campus is seen to become a living laboratory and a playground inspiring the inventiveness and enabling to test the new ideas and concepts effectively.

Aside of the versatile education-focused technologies (e.g., teleconference and online-learning), a smart campus implies the presence of multiple low-energy devices, such as the various sensors and actuators. The state-of-the-art approach to enable their connectivity is primarily based on the use of the Low Power Wide Area Network (LPWAN) [2] and Machine-Type Communications (MTC) [3] technologies. The former is typically associated with the technologies operating in the unlicensed frequency bands, while the MTC is often used as a synonym for the connectivity solutions for IoT standardized by the $3^{\text {rd }}$ Generation Partnership Project (3GPP) - i.e., the Narrow-Band IoT (NB-IoT) and LTE-M.

Over the past few years, multiple scholars focused on instrumenting and studying real-life large-scale deployments based on the LPWAN technologies. The industrial monitoring application [4], remote healthcare use cases [5], a smart lighting system [6] as well as a smart traffic light for congestion monitoring [7], real-time monitoring in power distribution grids [8] based on LPWAN have already been implemented, and their network performance was investigated. Moreover, the real estate monitoring use cases inside the university premises, providing the very basis of the smart campus, were addressed as well [9].

Nevertheless, none of these efforts, to the best of our knowledge, presents the empirical analysis of the NB-IoT and its performance in the context of the smart campus applications. With the guaranteed QoS and agreed Service Level Agreements (SLAs) featured by NB-IoT [10], IoT can now be considered for the use cases, which are more demanding for the connectivity performance [11]. Another principal difference between our work and the previous studies is the novel deployment and business model of the NBIoT network under research. The NB-IoT network, which is investigated in this paper, is owned and controlled not by a traditional telecom operator, but by the university itself. Thus, the university plays a role of a micro-operator [12] - a new entity that is expected to appear in the post-4G landscape [13]. Specifically, by moving the communication infrastructure closer to the users one enables higher connectivity performance and can fight against the radio channel attenuations, which are especially significant indoors. However, traditional commercial telecom operators often have no will, or no technical or legal capability to deploy their equipment indoors. In such a case, their role of connectivity providers may be taken by other entities, e.g., the land/building owners, tenants, or dedicated service providers. These connectivity providers are refereed to as the microoperators and are expected to play an important role in the $5 \mathrm{G}$ and future $6 \mathrm{G}$ connectivity provision, and, especially, with respect to the smart campus initiative. Importantly, in campus environment, the microoperator not only provides the connectivity service, but also supports teaching and research.

In this paper, we assess the feasibility and evaluate the key application-level communication performance indicators (i.e., the reliability, latency, and throughput) of a smart-campus 


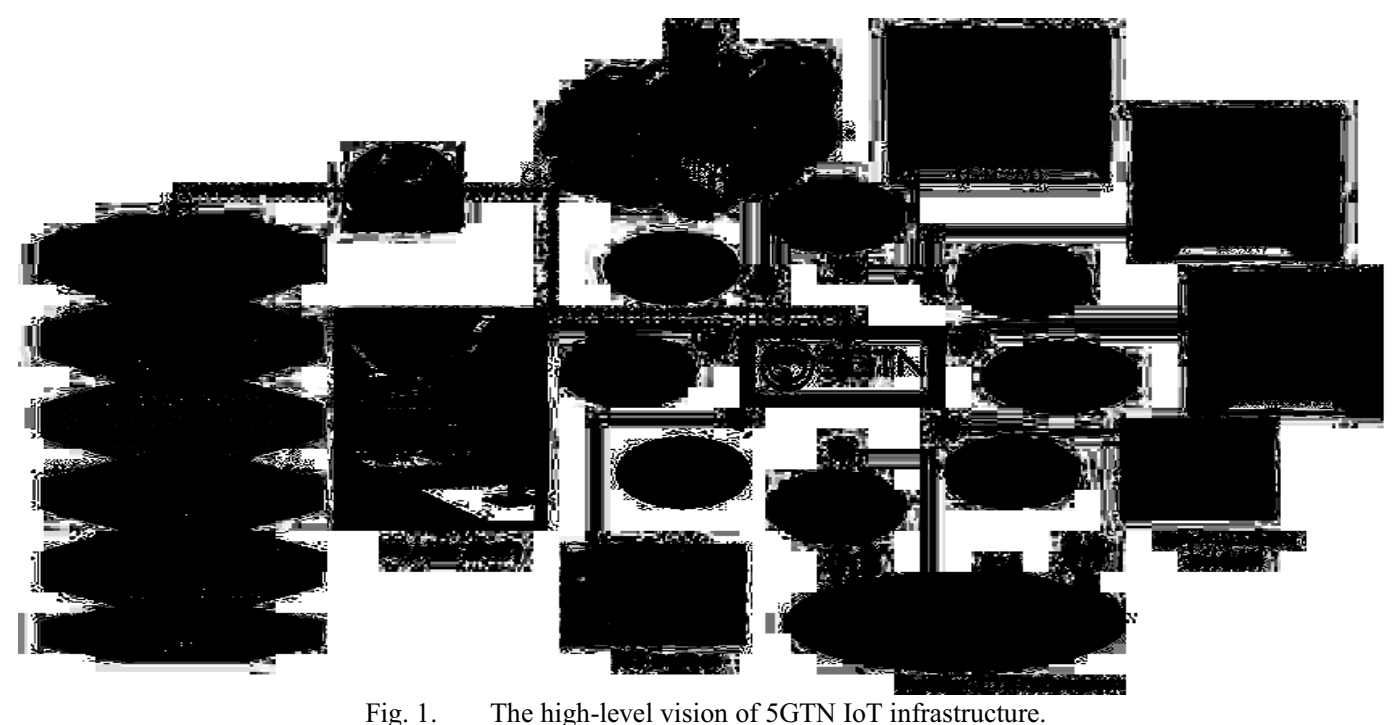

micro-operator-based NB-IoT network. Given the specifics of the architecture of the University of Oulu campus, in which all the buildings with the total net floor area $188600 \mathrm{~m}^{2}$ [14] represent one single interconnected indoor environment, the current study reports the most extensive experimental study of the NB-IoT indoors to this day. Aside from this, we report the results providing operational insight and empirical analysis of the technology with respect to the following aspects:

- revealing an initial experimental behavior of the NB-IoT network in actual operation;

- addressing the NB-IoT capability to sustain different indoor IoT applications and use cases in terms of reliability, throughput and latency;

- discussing the aspects, which limit the performance matrices, especially for smart campus use cases.

The paper is organized as follows. In Section II, we briefly discuss the basics of NB-IoT technology. In Section III, we present the 5GTN and explain our experimental environment. Section IV details the results of our experimental campaign. In Section $\mathrm{V}$, we summarize our results, speculate about the potential use cases for NB-IoT in smart-campus context and discuss some of our experiences while acting as an NB-IoT micro-operator. Finally, Section VI provides conclusions and briefly discusses further work directions.

\section{TECHNICAL ASPECTS OF NB-IOT}

The initial standard for NB-IoT was released by 3 GPP as a part of release 13 [15]. The standard defines three deployment alternatives for NB-IoT. The first one is the standalone operation mode operating on the radio spectrum, which has been previously not used or has been freed by GERAN systems [16] taken out of use. The two other deployment modes, i.e., the guard-band and in-band, allow NB-IoT to be used in parallel with the Long-Term Evolution (LTE) access networks. The guard-band mode utilizes the unused resource blocks within an LTE carrier's guard-band, and the in-band deployment implies the sharing of time-frequency resources between LTE and NB-IoT.

The NB-IoT (NB1) network operates on bands 1, 3, 5, 8, $12,13,17,19,20,26$, or 28 [16] and the logic of NB-IoT device operation is very similar to that of LTE. First, an NBIoT device searches for the active cells. The device first has to detect the synchronization signals (Narrowband Primary

Synchronization Signal (NPSS) and Narrowband Secondary Synchronization Signal (NSSS)) and then receive the Master Information Block (MIB) and a number of System Information Blocks (SIBs). MIB and SIBs carry the key information about the cell and the network and are sent in the Narrowband Physical Broadcast Channel (NPBCH) and Narrowband Physical Downlink Shared Channel (NPDSCH), respectively.

In order to establish a link, an NB-IoT device starts by sending a random-access preamble in the Narrowband Physical Random Access Channel (NPRACH) during the specially-allocated random access time window. The timings and the number of the preamble repetitions are defined based on the device's Coverage Enhancement (CE) level, which is determined from the Reference Signals Received Power (RSRP). In the case, if multiple devices attempt to establish a connection to the network simultaneously and select the same random preamble, some of them can undergo an RA failure.

If a Base Station (BS) receives and correctly decodes the random access preamble, the communication continues using the Narrowband Uplink Shared Channel (NPUSCH), NPDSCH, and Narrowband Physical Downlink Control Channel (NPDCCH), the resources in which are allocated by the BS. The NPDCCH is used by the BS to allocate the resources for uplink transmission in NPUSCH to a device or to signalize a device when downlink data for it is transmitted in NPDSCH.

The NPUSCH is used to convey the uplink traffic and provide the acknowledgments. The power allocation for NPUSCH transmission relies on the estimated path loss of the radio condition for that serving cell. The open-loop uplink power control without feedback mechanism reduces the complexity and signalling.

To achieve long communication range the NB-IoT uses several mechanisms. The first one is the control over the uplink transmit power (which can reach $23 \mathrm{dBm}$ at maximum). Next, a cell may support up to two CE levels in addition to the default one. If the signal level falls beyond a particular threshold, the device may switch to the next CE level, thus increasing the number of radio-symbol repetitions.

The three key mechanisms enabling power savings for NB-IoT are: 


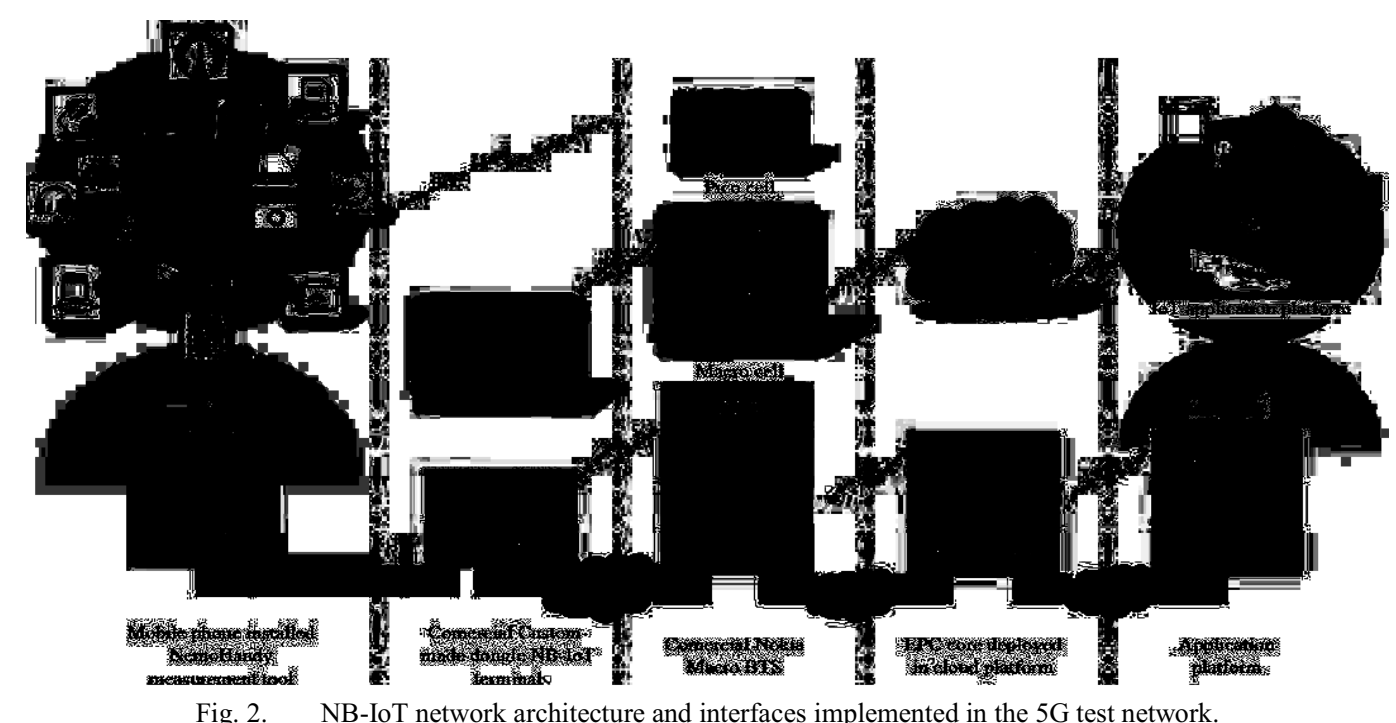

Fig. 2. NB-IoT network architecture and interfaces implemented in the $5 \mathrm{G}$ test network.

- The extended Discontinuous Reception (eDRX), which enables a device to stop monitoring the control channels periodically.

- The Power Saving Mode (PSM), which enables a device in idle state to disable the radio and enter low-power sleep while retaining registration in the network.

- The Release Assistance Indication (RAI), enabling a device to signalize that it does not expect further data transfers right now and request closing the connection.

\section{5G TEST NETWORK AND EXPERIMENTAL SETUP}

\section{A. $5 G$ Test Network Background and Capabilities}

The 5G Test Network (5GTN) has been deployed at the University of Oulu in order to enable an open access live network for the research and development (R\&D) activities. The network is the principal infrastructure component of the 6G Flagship [17] program and is controlled and operated by the University of Oulu, and the Centre for Wireless Communications specifically, acting as the micro-operator for the $5 \mathrm{GTN}$. The $5 \mathrm{GTN}$ is employed for versatile education, research, and development actions by the university itself, and the large-scale testing by the industry consortium partners affiliated with 5GTN and 6G Flagship.

Fig. 1 depicts the high-level vision of 5GTN IoT infrastructure. The IoT-enabled platform is provided as Infrastructure as a Service (IaaS), Network as a Service (NaaS), and Platform as a Service (PaaS) for application developers, device manufacturers, and university researchers [11]. As one can see from Fig. 1, 5GTN supports multiple radio access technologies, including, e.g., LTE, LoRaWAN, and even 5G. Nonetheless, in this study, we focus specifically on the connectivity of IoT-enabled devices through NB-IoT.

This is worth noting that the focus of 5GTN is not limited to only smart campus applications or use cases. By facilitating the platform openness, 5GTN intends to support different prospects of IoT for Smart Health, Robotics, Wearable devices, Smart Education, Smart Green House, etc.

\section{B. NB-IoT in 5GTN: experimental and measurement setup}

In what follows, we explain our experiment setup starting from the level of a device. The end-to-end measurement setup is illustrated in Fig. 2.
The device used for testing is the kDC-5737 NB-IoT wireless data terminal from Knowyou Technologies [18]. The device is a custom-made multi-band dongle with a Qualcomm chipset, supporting bands 1, 2, 4, 5, 7 for LTE as well as band 28 for NB-IoT. The terminal has a rubber bar antenna with an antenna gain of $1.9 \mathrm{dBi}$. The NemoHandy [19], a handheld wireless measurement tool, is used to perform the measurements. To ensure mobility during the measurements, the NB-IoT terminal (dongle) is coupled with an Androidbased phone with the NemoHandy application running. Both the technology (NB-IoT) and the frequency band are locked during the experiments.

The test device is connected to a Radio Access Network (RAN), composed of a Nokia Macro BTS delivering an LTE (band 7 FDD) and an NB-IoT (band 28 FDD) cells. The BS creates two on-air cells, each of which is served by a sectoral antenna with $14.5 \mathrm{dBi}$ gain. Note that the NB-IoT infrastructure of $5 \mathrm{GTN}$ also includes several pico BSs, which are deployed around the campus and operate in bands 1 and 7 , and which have not been used for this measurement campaign. The BS's maximum transmit power is set to $39 \mathrm{dBm}$ and it is configured with downlink and uplink EARFCN 9385 (775.50 MHz) and $27385(720.50 \mathrm{MHz})$, respectively. The NB-IoT $\mathrm{UL} / \mathrm{DL}$ channel bandwidth is $0.2 \mathrm{MHz}$ and the expected cell span is about $15 \mathrm{~km}$. For our experiments the cells are configured with only one CE level with $16 \mathrm{RACH}$ preamble repetitions and $160 \mathrm{~ms} \mathrm{RACH}$ period. The S1 interface from the LTE BS towards IP layer connectivity, i.e., the backhaul, is terminated locally to a Cloud Core EPC featuring NB-IoT.

The measurements were taken indoor inside the University of Oulu while connected to a radio cell in which the antenna beam is directed towards North of the faculty of ITEE (see Fig. 3) and the antenna is mounted at a tower with a height of around $24 \mathrm{~m}$. The 23 indoor reference points were selected on the 1st floor of the University of Oulu, Linnanmaa campus. The individual locations of these points are marked along with the respective RSRP values on the campus map in Fig. 3.

For measuring the radio-channel related parameters and the Round-Trip Time (RTT), at least 50 ping requests, 32-byte each, were sent using the NemoHandy software to a test server. The server was located within 5GTN at the very edge of the network. To measure the throughput, we performed iperf test in Nemo outdoor application [19]. For the test, the configuration parameters were set as follows: 


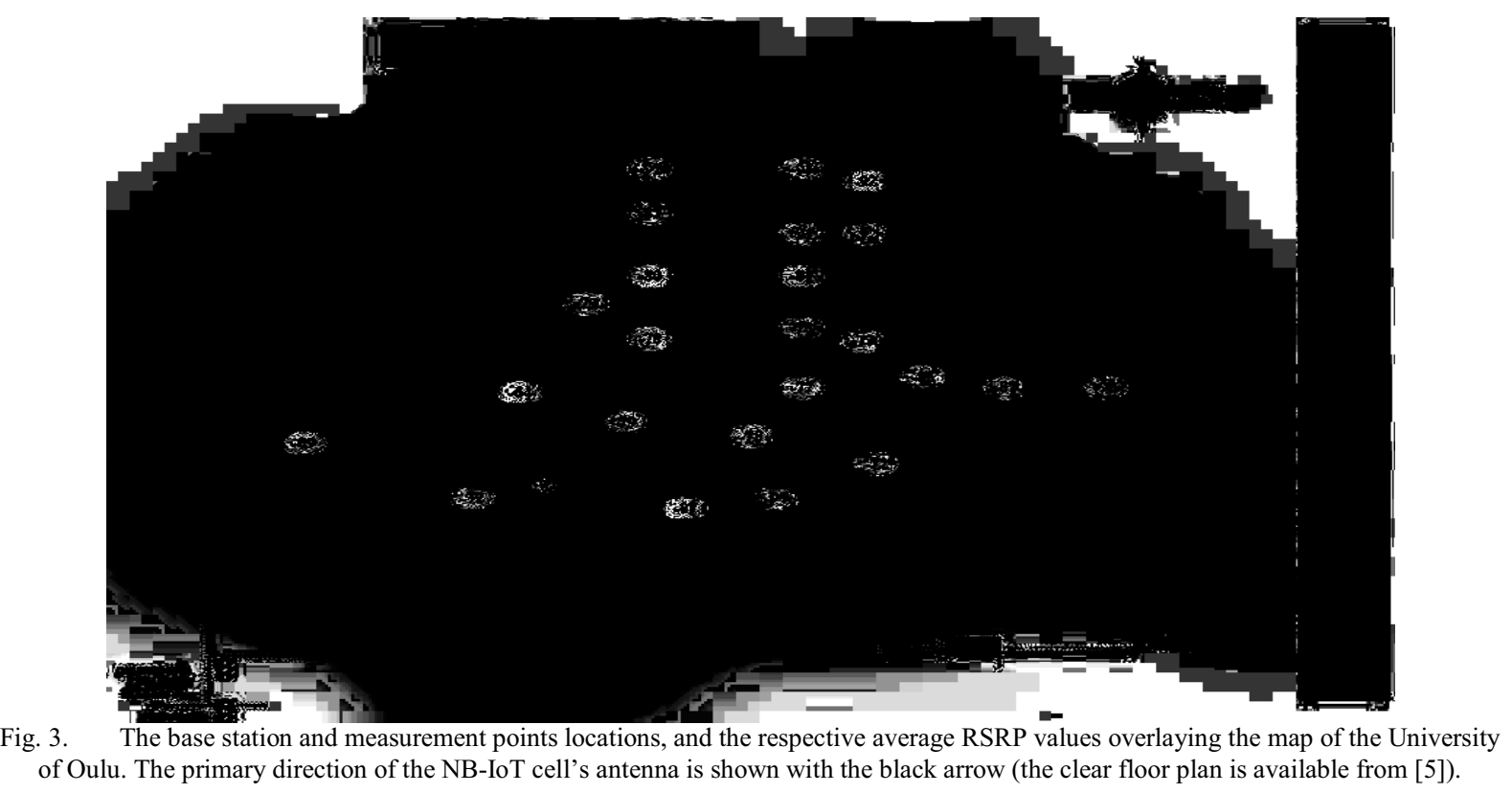

- UDP buffer size 8 kbytes

- UDP datagram size 1470 bytes

- UDP bandwidth $200 \mathrm{kbps}$

After activating the data session for NB-IoT terminal, the UDP data were sent to the same server, where the ping requests were addressed. The tests were done for 60 seconds on each measurement point. The NPUSCH and NPDSCH throughputs were recorded, and the maximum values were taken for each test point.

\section{EXPERIMENTAL RESULTS}

Fig. 3 provides an insight into the coverage of the NB-IoT macro BS of 5GTN. The figure illustrates the position of the BS and the test-points with average RSRP for each of them, which overlay the campus map of the University of Oulu, Linnanmaa campus. The NB-IoT macro has a 4-port dualpolarization antenna with a half-power beamwidth of $65^{\circ}$ and an adjustable electrical down tilting of $2-16^{\circ}$. As far as the horizontal pattern of the antenna is concerned, it has azimuth beamwidth of $62 \pm 3.6^{\circ}$, the front-to-back ratio for total power $\pm 30^{\circ}$ greater than $24 \mathrm{~dB}$, the cross polar discrimination at boresight greater than $21 \mathrm{~dB}$ and azimuth beam port-to-port tracking less than $2.0 \mathrm{~dB}$. Furthermore, the vertical beam pattern has an elevation beamwidth of $13.2 \pm 0.7^{\circ}$, tilt accuracy less than $0.6^{\circ}$, first upper side lobe suppression greater than $14 \mathrm{~dB}$ and an upper sidelobe suppression, $20^{\circ}$ sector above main beam greater than $14 \mathrm{~dB}$. [11]. In the deployed macro cell the directions of the antenna radiation beam is $2400 \mathrm{~m}$ towards the North and the beam width is 1000 $\mathrm{m}$ from east to west approx. Figs. 4 and 5 detail these results further by presenting the RSRP, transmit power, SNR, and the estimate of the path loss for each of the test points, sorted by their Euclidian distance to the BS.

From the presented results, one can see that despite being located on the ground floor, all the test points are served by the $5 \mathrm{GTN}$. For all the test points, the SNR ranged from 10 to $20 \mathrm{~dB}$, and the path loss was below $120 \mathrm{~dB}$. For this reason, as can be seen from the chart, the transmit power used by the NB-IoT terminal was between -25 and $10 \mathrm{dBm}$. Note that the maximum transmit power for the Class NB1 device is 23
$\mathrm{dBm}$, and the maximum coupling loss for NB-IoT is expected to reach $164 \mathrm{~dB}$ [16]. Therefore, as one can see, having over $40 \mathrm{~dB}$ "reserve," the network has substantial extension capabilities. Nonetheless, one can see that due to the specifics of indoor radio signal propagation (affected, e.g., by the blockage, fading, reflection, scattering, etc.), not always the short Euclidian distance between a test point and the BS resulted in high RSRP. Another reason for this is the directivity patterns of the BS's antennas.

Next, we characterized the key application-level performance metrics: the UL/DL throughput and the latency. These results are presented in Figs. 6 and 7, respectively. The markers denote the UL/DL throughput and round-trip latency for each test point, sorted based on their Euclidian distance to the BS. The trendlines computed based on the moving average, are also presented on the figures to reveal the trends.

The presented results reveal that during the experiments the NPUSCH and NPDSCH throughput ranged from 11.31 kbps to $17.44 \mathrm{kbps}$ and from $0.71 \mathrm{kbps}$ to $9.52 \mathrm{kbps}$, respectively. One can see that the results for the various test points can differ drastically. To give an example, the throughput of $15.91 \mathrm{kbps}$ in NPUSCH and $9.52 \mathrm{kbps}$ in NPDSCH (i.e., maximum) were observed from a test point located in close to line-of-sight featuring RSRP of $-78 \mathrm{dBm}$. Meanwhile, for another point with the RSRP of $-64 \mathrm{dBm}$, the NPUSCH and NPDSCH throughputs were $16.23 \mathrm{kbps}$ and $0.80 \mathrm{kbps}$, respectively. On the other hand, for the test point with one of the lowest RSRP of $-92 \mathrm{dBm}$, the uplink (maximum) and downlink throughputs were $17.44 \mathrm{kbps}$ and $0.95 \mathrm{kbps}$, respectively. These results are rather surprising for us, and in the future, we plan to execute additional measurements to study this anomaly.

The results for RTT and the RACH access delay, which denotes the time period from transmitting msg1 to receiving msg4 as a part of the contention-based random-access procedure, are presented in Fig. 7. As one can see, for the majority of the points, the RTT was in the order of $2400 \mathrm{~ms}$, and the RACH access delay was $173 \mathrm{~ms}$ on average. Note, that since the RTT counting is started from msg1, only a single coverage extension level is enabled, and all the points are in decently good channel conditions, in case if no packets are lost 

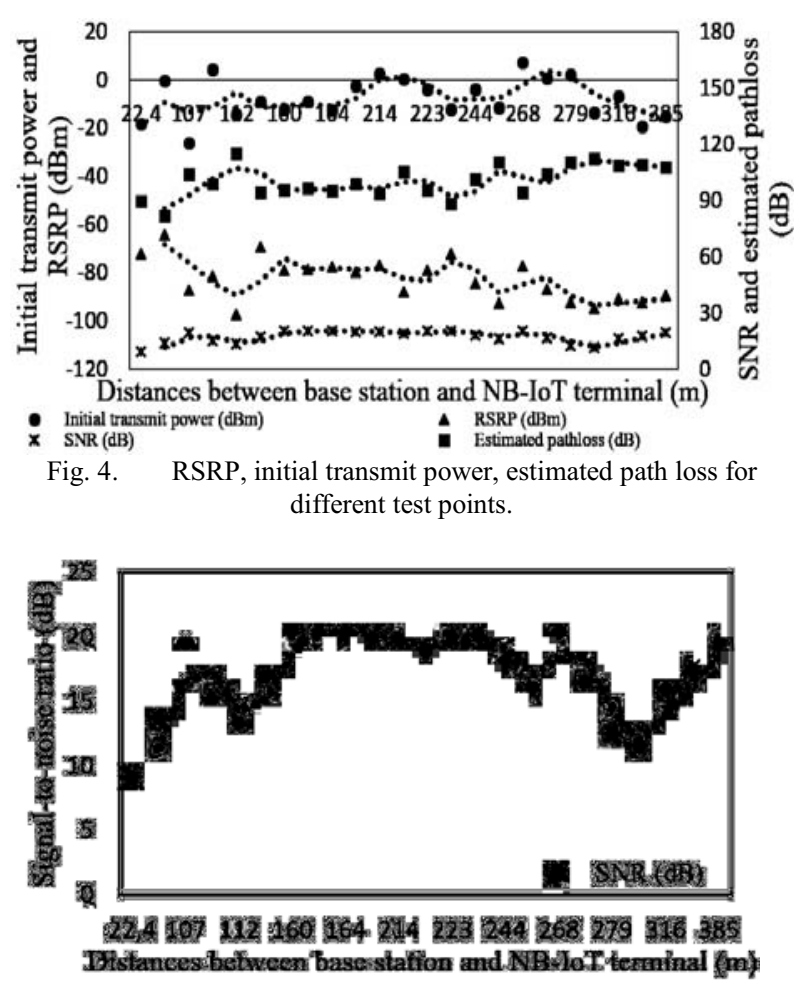

Fig. 5. Signal-to-noise level for different test points.

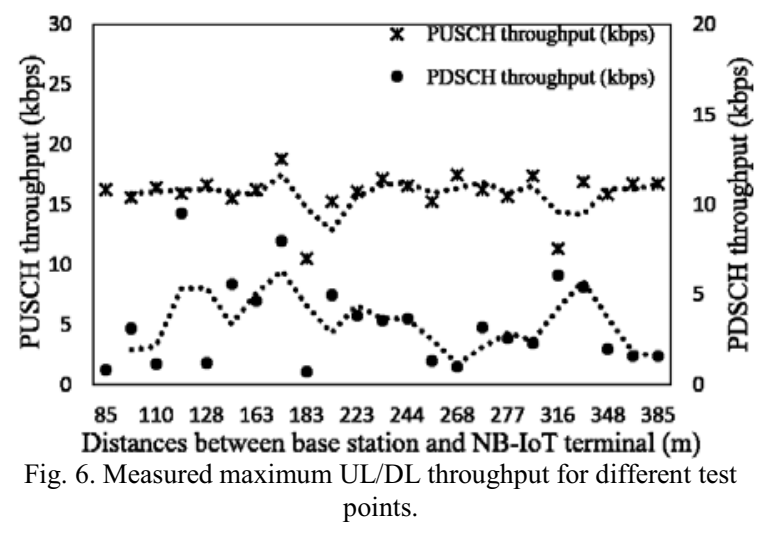

and the resources for uplink and downlink transmissions are available, the RTT is not be affected significantly from the distance between the BS and a test point. However, there were two test locations, for which both RTT and RACH access delay were substantially higher. The more detailed study of the experiment logs showed that when located in these points, the test device experiences substantial block losses (the block error rate was $33-50 \%$ ) and often had to transmit preamble more than once to access the channel. This, apparently, required time. Meanwhile, as this can be seen from Figs. 4 and 5, the SNR and RSRP in these test locations were quite high and did not differ substantially from that in the other test points. This can also be seen from the charts that the time for RACH composed only about $10 \%$ of the total RTT time. The maximum time for waiting for the next $\mathrm{RACH}$ window was a mere $160 \mathrm{~ms}$. Given that the test server was located within $5 \mathrm{GTN}$, its response time was within several dozens of milliseconds. Therefore, we expect that most of the delay is caused by resource allocation and communication in NPUSCH and NPDSCH. One of the possible reasons for this is the in-band deployment of NB-IoT within $5 \mathrm{GTN}$, requiring

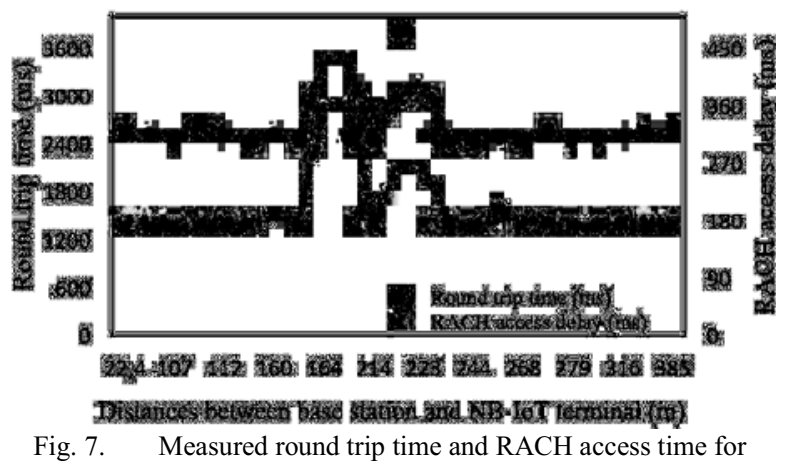
different test points.

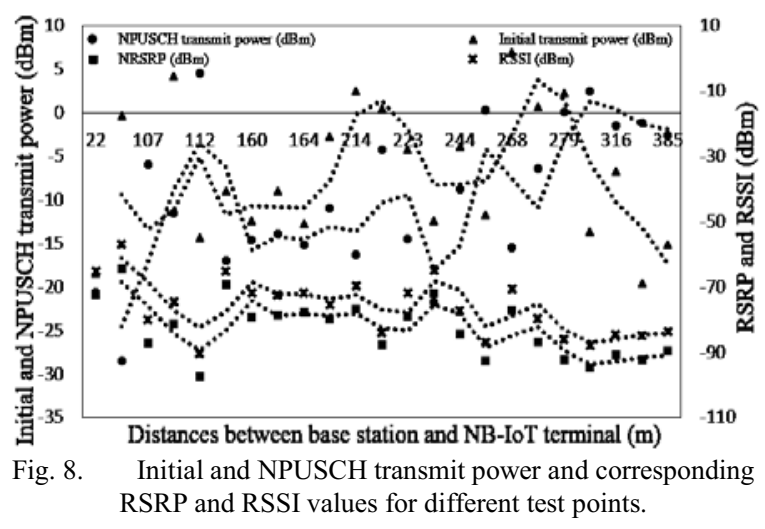

it to share the resources with LTE. The allocation of the resources to the conventional LTE may also be one of the reasons for the variations of the throughput observed during our experiments.

Finally, Fig. 8 demonstrates the dynamics of the transmit power control of the NB-IoT terminal, along with the measured RSRP and RSSI. The transmit power is one of the parameters, which contributes to the energy consumption of an NB-IoT device, and the mechanisms for controlling which differ for NPRACH and NPUSCH (see [20]). As one can see, for most of the test locations, the transmit power was below than $-2 \mathrm{dBm}(0.63 \mathrm{~mW})$.

\section{DISCUSSION AND LESSONS LEARNED}

As a micro-operator [21], the 5GTN provides a diverse connectivity infrastructure within a limited local area, including the university campus and a few other selected locations in the city of Oulu. Importantly, today the connectivity services are required not only by the people, but also by the versatile machines, ranging from tiny sensors to drones, and even connected vehicles. The NB-IoT technology is expected to play an important role in enabling the mass connectivity for low-end machines. Therefore, in this paper, we investigated the performance of a single-BS microoperator NB-IoT deployment. The key measurement results are as follows:

- $\quad$ NB-IoT demonstrates quite good coverage indoors, even for very extensive indoor environments.

- The application-layer latency observed in our measurements was in the order of seconds. The uplink throughput for most of the points was in the order of 15 kbps, but the downlink varied a lot - from hundreds of bps to ten kbps. 
- In most cases, the transmit power of NB-IoT devices was below $0 \mathrm{dBm}$.

Importantly, all these have been achieved with a single BS. Given these results, we expect that NB-IoT may play an important role in enabling delay-tolerant applications for devices with low traffic. These include, e.g., the various noncritical metering and actuating, as well as tracking applications. In the context of a smart campus, there are a plethora of use cases, which fit these. To name just a few: the measuring of lecture rooms/laboratories/restaurants/parking lots occupancy; the tracking of measurement tools and other properties; the remote control of lights and other non-critical infrastructure (e.g., the displays). In this context, the microoperator approach, when the university owns and manages the infrastructure providing it also as a service to other service providers, is very attractive.

Meanwhile, the key challenges, which we have faced implementing this concept, are related to (i) negotiating and obtaining the needed licenses and (ii) managing and configuring the network. The former is mostly a political issue, which requires goodwill and partnership from both the telecom operators and the governmental authorities. The latter originates from the complex nature of the NB-IoT technology and a great number of parameters, affecting its operation. To give an example, we observed that the application-level performance for latency and energy consumption is strongly affected by the number of coverage extension levels and their configurations (i.e., the RSRP thresholds, RACH periods, number of repetitions). Another parameter, which affects the energy consumption and throughput/latency is the inactivity timeout, which determines the time period after which a device gets disconnected from an NB-IoT network if no communication goes on. Decreasing this timeout enables energy savings, but, as we have observed, may prevent a device from connecting to the network if the time for connection authorization will exceed the timeout. When this comes to the technical aspects, we found the NB-IoT technology and the hardware/software used to be mature enough not to feature any major technical issues.

\section{CONCLUSIONS}

In this paper, we discussed the deployment and reported the practical performance of a single-cell NB-IoT microoperator deployment as a part of the 5GTN. Our results demonstrate that NB-IoT technology is a viable connectivity solution for various non-critical machine-based applications deployed indoors. To the best of our knowledge, this paper presents one of the first attempts to extensively investigate the NB-IoT performance indoors. Therefore, the presented results may be interesting for various stakeholders. Also, in the paper, we highlighted some practical lessons learned during the deployment and management of an NB-IoT campus network acting as an IoT micro-operator.

In future, the 5GTN network will be substantially extended by adding the new connectivity solutions, as well as by bringing into it more applications. Also, we intend to continue the research activities using the network, focusing on the effect of the different network parameters on the performance, investigating the detected anomalies further, and addressing the aspects, which we have not considered in this work - e.g., the energy efficiency and the scalability.

\section{ACKNOWLEDGMENT}

This work has been performed utilizing the NB-IoT network offered as a service by $5 \mathrm{GTN}$ at Centre for Wireless Communications, University of Oulu. This research has been financially supported by Academy of Finland 6G Flagship (grant 318927).

\section{REFERENCES}

[1] S. Du, F. Meng and B. Gao, "Research on the Application System of Smart Campus in the Context of Smart City," in Proc. Intern. Conf. on Inf. Tech. in Medicine and Education, 2016, pp. 714-718.

[2] K. Mekki, E. Bajic, F. Chaxel, and F. Meyer, "Overview of Cellular LPWAN Technologies for IoT Deployment: Sigfox, LoRaWAN, and NB-IoT," in Proc. IEEE Intern. Conf. on Pervasive Computing and Communications Workshops, 2018, pp. 197-202.

[3] S. Böcker, C. Arendt, P. Jörke, and C. Wietfeld, "LPWAN in the Context of 5G: Capability of LoRaWAN to Contribute to mMTC," in Proc. $5^{\text {th }}$ World Forum on IoT, 2019, pp. 737-742.

[4] P. Sommer, et al., "Low-Power Wide-Area Networks for Industrial Sensing Applications," in Proc. Intern. Conf. on Industrial Internet, 2018, pp. 23-32.

[5] J. Petäjäjärvi, K. Mikhaylov, R. Yasmin, M. Hämäläinen, and J. Iinatti, "Evaluation of LoRa LPWAN technology for Indoor remote health and wellbeing monitoring," Intern. Journal Wireless Inf. Networks, vol. 24, pp. 153-165, 2017.

[6] Y. S. Chang, Y. H. Chen, and S. K. Zhou, "A smart lighting system for greenhouses based on Narrowband-IoT communication," in Int.. Microsyst., Pack., Assembly and Circ. Tech. Conf., 2018, pp. 275-278.

[7] R. F. A. M. Nor, F. H. K. Zaman, and S. Mubdi, "Smart traffic light for congestion monitoring using LoRaWAN," in Proc. IEEE Control and Syst. Graduate Research Colloquium, 2017, pp. 132-137.

[8] G. d. Campo, et al., "Hybrid LPWAN Communication Architecture for Real-Time Monitoring in Power Distribution Grids," in Proc. World Forum on IoT, 2019, pp. 920-924.

[9] R. Yasmin, J. Petäjäjärvi, K. Mikhaylov and A. Pouttu, "Large and Dense LoRaWAN Deployment to Monitor Real Estate Conditions and Utilization Rate," in Proc. Intern. Symposium on Personal, Indoor and Mobile Radio Commun., 2018, pp. 1-6.

[10] B. Martinez, F. Adelantado, A. Bartoli and X. Vilajosana, "Exploring the Performance Boundaries of NB-IoT," IEEE IoTJ., vol. 6, no. 3, pp. 5702-5712, 2019.

[11] 5GTN- 5G Test Network. [Online]. Available: http://5gtn.fi/.

[12] J. S. Walia, H. Hämmäinen, and M. Matinmikko, "5G Micro-operators for the future campus: A techno-economic study," in Internet of Things Business Models, Users, and Networks, 2017, pp. 1-8.

[13] M. Latva-aho and K. Leppänen, "Key Drivers and Research challeges for 6G Ubiquitous Eireless Intelligence," 6G Flagship, University of Oulu, Finland, 6G Research Visions 1, 2019. [Online]. Avaialble: https://ytd2525.files.wordpress.com/2019/09/isbn9789526223544.pdf

[14] Google. "Oulu: Linnanmaa campus." [Online]. Available: https://sykoy.fi/en/campus/oulu-linnanmaa-campus/.

[15] 3GPP. "LTE; Evolved Universal Terrestrial Radio Access (E-UTRA); User Equipment (UE) radio transmission and reception," 3GPP TS 36.101, version 13.6.1, release 13 .

[16] 3GPP. "Evolved Universal Terrestrial Radio Access (E-UTRA); Physical layer procedures," 3GPP TS 36.213,version 15.2.0,release 15.

[17] 6G Flagship. [Online]. Available: https://www.oulu.fi/6gflagship/.

[18] Knowyou Technologies, "KDC-5737 NB-IoT Wireless Data Terminal User Manual.” [Online]. Available: https://usermanual.wiki/KnowyouTechnologies/KDC-5737.

[19] Nemo. "NemoHandy Handheld Measurement Solution." [Online]. Available: https://www.keysight.com/.

[20] 3GPP. "LTE; Evolved Universal Terrestrial Radio Access (E-UTRA); Medium Access Control (MAC) protocol specification" 3GPP TS 36.321, version 13.0.0, release 13 .

[21] M. Matinmikko, M. Latva-aho, P. Ahokangas, and V. Seppänen, "On regulations for 5G: Micro licensing for locally operated networks," Telecommunications Policy, vol. 42, no. 8, pp. 622-635, 2018. 